\title{
Multi-criteria Optimal Design of Cable Driven Ankle Rehabilitation Robot
}

\author{
P. K. Jamwal, S. Q. Xie, K. C. Aw and Y. H. Tsoi \\ University of Auckland \\ New Zealand
}

\section{Introduction}

An ankle rehabilitation robot has been conceptualized and designed to realize the range of motion, muscle strengthening and proprioception training exercises for ankle joint. The robotic device is intended to help patients and therapists in their cooperative efforts for the treatment of impaired ankle joint as a result of injury or stroke. After analyzing the ankle joint anatomy and its motions, a parallel mechanism is proposed for the robot. To mimic the human ankle joint and its muscles actuation, the proposed robot uses artificial air muscles configured in a fashion close to the actual muscle arrangement. The apparent advantages of the proposed robot over the existing ankle rehabilitation parallel mechanisms have been emphasized. As a matter of fact, the performance of parallel robots greatly depends on their dimensions and the configuration of their actuators. Thus to explore the potential of these robots, it is essential to obtain a set of kinematic parameters, leading to optimal robot performance. To achieve this, robot designs need to be optimized on the basis of performance indices such as, workspace, condition number and Euclidean 2-norm of actuator forces, under various operational constraints. The performance criteria and the constraints are discussed in detail to justify their influences on the robot design. The existing Multi-objective Optimization Approaches (MOA) e.g. weighted formula approach, population based approach and Pareto optimal approach have been discussed. The algorithm used in this chapter is based on genetic algorithms and attempts to draw advantages of the weighted formula and the Pareto optimal approaches simultaneously for the optimization of robot design. The results obtained from the optimization are discussed and important inferences for further work are drawn.

\section{Rehabilitation and Robotics}

Rehabilitation in a broader sense means a practice by which any form and grade of human physical disorder can be reinstated. The disorder could be the result of an injury or a stroke. Conventionally, to restore range of motions and strength of limbs, rigorous and repetitive exercises are performed under the supervision of a therapist. These exercises over the time improve motor functions by enhancing neuro-plasticity and neuro-recovery at the affected limbs. Apparently during a rehabilitation treatment, cooperative efforts of therapist and 
patient are required over prolonged sessions of treatment in a clinic. Moreover the patient is required to continue the prescribed exercises at home for a speedy recovery. It has been documented (Krebs et al., 2003) that using conventional way of treatment the recuperation is slow and sometimes continues for more than a year. The patient, the therapist and the rehabilitation process suffers from the drawbacks of conventional treatment. Patients have to travel in their disabled state to attend the clinical sessions which is undesirable especially when they have lower limb injuries. Treatments in the rehabilitation clinic are costly and time consuming, considering the travel time and the waiting time of patients. Furthermore, exercises advised by the therapist are monotonous and lacks motivation, hence resulting in inadequate improvement. Similarly the therapist has to perform strenuous and repetitive efforts with the patients and thus he can only attend a limited number of patients in a day. Due to lack of documented history of the patient's improvement, therapists normally advise further treatment based on their own perception which adds to the undesired subjectivity. Robotics can play an important role in the process of rehabilitation by assisting the therapist and the patient. While using the robot, the patient doesn't get tired of moving his ankle, as is now being moved by the robot for the range of motion exercises. Further to make exercises more interesting and motivating certain visual and haptic effects can be appended with the robot. Using a personal computer, the therapist can establish a remote connection with the patient's robot and get the required information about his progress. Similarly the patient can also receive instructions from therapist by staying at home. Rehabilitation process can also be improved by acquiring progressive data of patient's improvement, which in turn can help the therapist to make systematic decisions on the choice of further exercises. Moreover the expert knowledge of the therapist can be incorporated in the robot controller to make it adaptive to different modes of exercises.

Rehabilitation robots are different (Tejima, 2000) from industrial robots in application and operation and hence special care must be taken in their design. Human augmented robots should be especially safe to use and must be user friendly in operation. This calls for ergonomic design and intelligent and adaptive robot controllers. Thus the design and control of these robots are challenging tasks requiring multi-disciplinary skills and in-depth knowledge of human joint anatomy and movements.

There are robotic devices currently in use such as MIT-MANUS for the upper limb rehabilitation (Krebs et al., 2003), LOKOMAT for gait training (Hesse et al., 2003) and Rutgers Stewart platform and other parallel robots (Dai et al., 2004) for ankle rehabilitation. However, the potential of robotics in rehabilitation has not been completely explored and key issues such as optimal design and intelligent and adaptive control, requires further research.

This chapter provides a discussion on the complexities of the ankle joint, its rehabilitation and challenges on the optimal design and development of a new parallel rehabilitation robot. Section 3 elucidates the anatomy, problems and physiotherapy of the human ankle joint along with a brief review on existing robotic devices and their shortcomings. A new wearable parallel robot which has been conceptualized to compensate the drawbacks of previous designs is proposed in Section 4 with brief discussion on its kinematic and geometrical modeling and the workspace analysis. The important design criteria and their significance are discussed in Section 5, followed by the design optimization problem formulation in Section 6. Section 7 investigates possible approaches to solving multi-criteria and multi-variable optimization problems. Genetic algorithm (GA) has been used to 
implement the proposed optimization scheme and hence the GA methodology and the key steps of the proposed algorithm are also explained in Section 7. Results obtained from our proposed algorithm are discussed along with some inferences in Section 8. Conclusions drawn and the future work are presented in Section 9.

\section{Human Ankle, its Problems and Physiotherapy}

\subsection{Ankle Complex}

The ankle is the most complex bony structure in the human skeleton (Dul and Johnson, 1985) and is a combination of two joints (Figure1). The first joint is called the ankle joint which is made up of three bones: the lower end of the tibia (shinbone), the fibula (the small bone of the lower leg) and the talus (the bone that fits into the socket formed by the tibia and the fibula). The talus sits on top of the calcaneus (the heel bone) and moves mainly in one direction. The ankle joint works like a hinge to allow foot to move up (dorsiflexion) and down (plantar flexion). The second joint is the subtalar joint, also known as the talocalcaneal joint and this is a joint of the foot. It occurs at the meeting point of the talus and the calcaneus. This joint is responsible for the inversion and eversion of the foot, but plays no role in dorsiflexion or plantarflexion of the foot. However it is very much a part of the ankle joint and thus can not be ignored.

There is one more joint called MTP (metatarsophalangeal) joint connecting fore and the rear with Calcaneus, Cuboid and Navicular bones as shown in Figure1. The raising and lowering motions of the Toe and the heel are achieved about this joint. In our study the ankle and the subtalar joints have been collectively considered as a spherical joint and are called ankle joint henceforth for simplicity. Since our study is limited to the ankle joint motions and not the fore foot motions, the MTP joint is not considered.

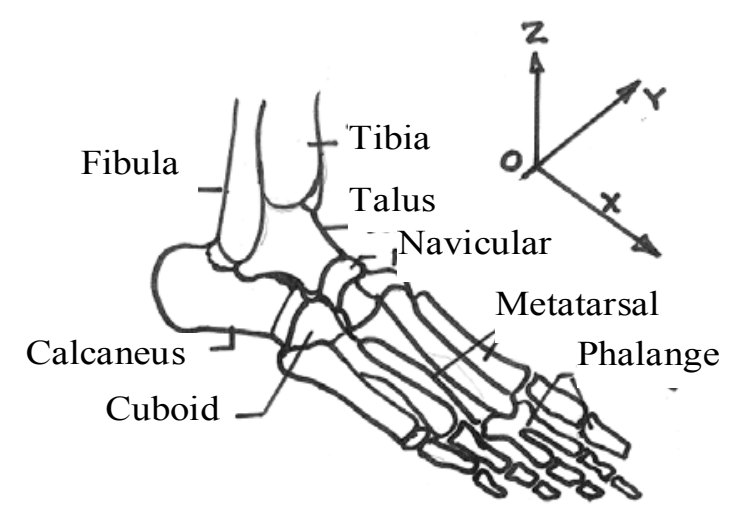

Fig. 1. Schematic of the Ankle and the sub talar joint 
There are ligaments on both sides of the ankle joint that hold the bones together and many tendons cross the ankle to help move the ankle and the toes. Ligaments connect bones to bones while tendons connect muscles to bones. The ankle joint is capable of rotations in all three planes (sagittal, frontal and transverse planes), sagittal plane is defined by $x$ and $z$ and movements in this plane occur around the $y$ axis as shown in Figure 1; transverse plane is defined by $x$ and $y$ and movements in this plane occur around the $z$ axis; and frontal plane is defined by $y$ and $z$ and movements in this plane occur around the $x$ axis. Various ankle movements (Siegler et al., 1988) and passive moment requirements (Parenteau et al., 1998) are summarized in Table 1.

\begin{tabular}{|c|c|c|c|}
\hline Axes & Name of the motion & Range of Motion & $\begin{array}{c}\text { Torque } \\
\text { Requirement } \\
(\mathrm{Nm})\end{array}$ \\
\hline $\mathrm{X}$ & Inversion & $14.5^{\circ}-22^{\circ}$ & 48 \\
\cline { 2 - 4 } & Eversion & $10^{\circ}-17^{\circ}$ & 34 \\
\hline $\mathrm{Y}$ & Dorsiflexion & $20^{\circ}-30^{\circ}$ & 50 \\
\cline { 2 - 4 } & Plantarflexion & $37^{\circ}-45^{\circ}$ & 50 \\
\hline $\mathrm{Z}$ & Adduction & $22^{\circ}-35^{\circ}$ & 40 \\
\cline { 2 - 4 } & Abduction & $15^{\circ}-25^{\circ}$ & 40 \\
\hline
\end{tabular}

Table 1. Approximate limiting values of range of motions and the passive moments at ankle joint.

\subsection{Ankle Injuries and Physiotherapy}

Ankle injuries (Dul and Johnson, 1985) are one of the most common injuries in sports and daily life. Youngsters are subjected to ankle injuries from sports and whilst carrying excessive load whereas children and the elderly gets them from walking on uneven surfaces and bone weakness. Non-functionality of ankle joint is also quite common in stroke surviving patient.

Common ankle injuries are sprain, strain and fracture. An overstretched muscle or tendon causes strain which is a mild injury. However if a ligament is overstretched it causes more serious injury called sprain which results in pain and joint non-functionality. Sometimes when a ligament is overstretched or broken it may pull off a piece of bone causing a fracture.

Primary treatment for ankle injuries (Dai et al., 2004) includes, rest, ice, compression and elevation (RICE) of the affected foot. Ice is used to reduce swelling, compression stockings are used to firmly support the ankle and foot and elevation helps to minimize further swelling. Surgical repair of the ankle ligaments is not required until the sprain in the ankle is recurrent. The primary treatment should be followed by some stretching and exercise therapy along with partial weight bearing to maintain mobility in the ankle. Achilles tendon is the strongest muscle of the body and is responsible for the calcaneus motion. It should be put in stretching exercise as soon as possible (within 48 to $72 \mathrm{hrs}$ ) after the injury to recover the range of motions (ROM). Once the ROM is achieved, strengthening of weakened muscles is essential for rapid recovery and is a preventive measure against further reinjury. As the patient achieves full weight bearing capability without pain, proprioceptive exercise is initiated for the recovery of balance and postural control using wobble boards. Finally, 
advanced exercises using uneven surface wobble board should be performed to regain functions specific to normal activities.

The ankle joint is an important joint in human skeleton since it is responsible to carry the body weight and maintain balance during gait. It is subjected to high impact forces which may be as high as several times of the body weight. It is a very strong joint with stiffer muscles and hence offers large resistive moments as mentioned in Table 1 . In the light of above facts, it can be concluded that a wearable robotic device for ankle rehabilitation should have high stiffness and higher payload capacity to realize required passive and resistive moments at the ankle joint. Moreover the robot should be light in weight so that the patient can comfortably wear it on his leg.

\subsection{Ankle Rehabilitation Robots}

For ankle rehabilitation, typical devices such as elastic bands, wobbles boards and foam rollers are in use, but they allow only simple and functional rehabilitation exercises. Commercially available rehabilitation units such as ARTU, Biodex and Pro-fitter can be used but they are expensive, function specific and are not versatile. Conversely, a robotic device can be constructed to implement complete rehabilitation program which includes $\mathrm{ROM}$, muscle strengthening and proprioception training. Physiotherapy requires painstaking repetitive movements of limbs about their respective joints and robotic machines are useful in such applications once appropriately programmed. Robotic devices can be programmed by a physiotherapist and exercise modes could be selected depending on the type of injury and patient's state of disability. Thus, task oriented training by supervised robotic systems is helpful to provide the patient with more useful exercises. Looking to the requirements for a robot specific to ankle treatments (as discussed in the previous section), it is realized that the parallel mechanism is a good choice owing to its high stiffness and payload capacity. Parallel robots normally have two platforms, a fixed platform (F.P.) and a moving platform (M.P.) connected together with rigid or flexible links or joints. In a recent development researchers have proposed some ankle rehabilitation robots based on parallel mechanisms. The previous designs have been studied critically to provide inputs for our proposed robot. In one of the earliest works, (Girone et al., 1999) proposed the "Rutgers Ankle" that uses a Stewart platform which can provide six degrees of freedoms (DOF). Double acting pneumatic cylinders are used as the actuators to move the platform to perform various ankle rehabilitation modes. The patient's foot is fixed firmly to the platform and assistive or resistive forces are applied for passive and active mode of exercises respectively. This platform has further been interfaced with the game-like virtual environments (Girone et al., 2000) to make the exercises more interesting and enjoying for the patient. The Rutgers Ankle was also used in the clinical trials for post-stroke rehabilitation (Deutsch et al., 2007) apart from sprained ankle treatment. In a recent development (Deutsch et al., 2007) have proposed a remote console which is a telerehabilitation system providing real time interaction between the patient, the robot and the therapist sitting remotely. Though the Rutgers Ankle is well developed and is being used in the clinical trials, the mechanism has not been thoroughly analyzed from the point of view of its workspace optimization and stiffness analysis. Moreover the patient's leg also contributes to the overall dynamics of the mechanism since it is not constrained and this fact has not been highlighted. The position of the ankle joint in the robot does not remain constant and there is a possibility of small shift in the ankle joint location causing inconvenience to the patient and inaccurate control. Since 
the ankle movements in most exercises require less than four DOF motions, (Dai et al., 2004) proposed a parallel robot for sprained ankle treatments using a three and four DOF parallel mechanism with a central strut. Kinematic and stiffness analysis has been carried out for the proposed mechanism. In particular they have used a central strut and analyzed three different types of parallel robots in the domains of stiffness. A single platform-based reconfigurable robot mechanism has been proposed by (Yoon et al., 2006). This robot design considers the MTP joint apart from the ankle joint motions and has less than six DOF motions. Since it is a reconfigurable robot, the same platform can be used for ROM, muscle strengthening and proprioception training. An impedance controller is proposed for this robot wherein the impedance parameters can be varied to accommodate different exercise modes. A 3-RSS/S parallel mechanism is proposed by (Liu et al., 2006) and the kinematic design of prototype has been validated using simulations.

So far most platform type devices require patient's foot to be placed on top of the platform, actuated from the bottom. There are two major issues with such a configuration, firstly, when the platform and the foot fixed to the platform are moved the position of the ankle joint keeps changing with respect to the ground. This inaccuracy in the position of the ankle joint leads to control errors which are difficult to comprehend. Secondly, the existing platforms have translational motion along with the rotation which causes shift in the patient's leg. Hence the dynamic model of the robot should include dynamic inertia of the patient's leg which can not be estimated. In the absence of an accurate dynamic model, large trajectory errors are expected. In (Girone et al., 2000) authors have proposed an Inside Track 3D tracker to measure the position and the orientation of patient's shinbone to avoid trajectory errors and prevent ankle movements beyond specified ROM. However movements of the shinbone relative to the ankle joint definitely causes discomfort to the patient as he is required to change his position intermittently.

To compensate these issues we have considered a new configuration of actuators for our proposed design which is similar to the actual muscular system in the human leg. The actuation of the end platform is performed using air muscles connected parallel to the patient's leg in the robot. Thus in our proposed robot the position of ankle joint and the leg remains stationary when the foot is moved in different exercise modes.

\section{Proposed Soft Parallel Robot for Ankle Rehabilitation}

\subsection{Soft Parallel Manipulators}

The parallel mechanisms can be classified as Rigid Parallel Mechanisms (RPM), if the links connecting the two platforms are rigid bodies and Soft Parallel Mechanisms (SPM) when the links are tendons or cables. There are certain problems using conventional RPM for the proposed wearable design of the robot. First of all, for a wearable robot the weight of the actuators should be kept low so that the patient can comfortably move his leg around with the robot. The RPM's use linear actuators which are heavy and rigid and hence can not be used in the proposed robot. Secondly the RPM uses spherical joints which results in the reduced ROM of the robot.

Soft parallel devices are very light in weight and has higher payload to weight ratio. Air muscle actuators along with the cable in the proposed robot weigh only 85gm for each link which is very low compared to the conventional linear actuators which weigh approximately $2500 \mathrm{gm}$. SPM has simpler dynamic model than their rigid-link counterparts 
because the inertias of their links (i.e., cables) can be ignored. Soft parallel robots are being used recently (Alp and Agrawal, 2002) in a variety of applications ranging from sophisticated medical and manufacturing applications to simple construction and shipment activities. However, wire flexibility of SPM poses some constraints on the workspace and the robot controllability and an extra variable called 'tensionability' is required to be considered during its kinematic design. Thus the design of SPM's is more critical and it is essential to select design parameters carefully to achieve controllability.

\subsection{Proposed Wearable Ankle Rehabilitation Robot}

This chapter proposes a wearable air muscle actuated SPM for ankle joint rehabilitation treatments. The robot is designed to provide three rotational degrees of freedom to the ankle joint. The device uses two parallel platforms, a fixed platform (FP) built-in with a leg support structure and a moving platform (MP). Air muscles are used as the actuators and are mounted on the leg support with their actuating end connected to MP through cables. These cables pass through sleeves provided in the FP. The leg support structure is light in the weight and can be fixed to the patient's leg using straps moving over the knee and fixed at thigh. MP remains below the foot and has a heel locator and straps to locate and fix foot. The moving platform of the robot is shaped to form a shoe of varying size and shape. This MP or the shoe is attached to the leg support using a special mechanism which provides three rotational degrees of freedom to the shoe and has its center of rotation coincident with the ankle joint. As the air muscles can only pull and can not push hence to maintain the tension in all the cables it is desired to have redundant actuation. In fact all the cable based parallel robots have redundant actuation (Pusey et al., 2003) which means the robot needs ' $(n+1)^{\prime}$ actuators to achieve ' $n$-dof' motion of the manipulator. Hence to obtain 3-dof from the robot we have used a set of four air muscles and four cables. Coordinated and antagonistic actuations of air muscles will ensure desired changes in the wire lengths and pose of the moving platform subsequently for a range of ankle exercises.

The proposed robot is required to perform specified motions in 3-D space. To accomplish this, the end effector is moved in the workspace along a predefined trajectory. The position and the orientation of the robot end effector at a specified workspace location can be obtained by controlling the displacements of the cables. This calls for a mathematical model of the robot which can define the relationship between end effector motions and the cable displacements in the domains of time and space. This mathematical model is called kinematic model and the derivatives of this model describes mechanics of the motion without taking forces into account. The forces and/or the torques are considered with the mechanics of motion in the dynamic analysis. The kinematic and the dynamic study are essential tools for the design of proposed robot. Kinematic model describes the position and orientation of the end effector with respect to the FP and the dynamic model relates the applied forces and/or torques to the resulting robot motions. The kinematic parameters of joints are of two types, fixed parameters and the variables parameters. Fixed parameters are the location of the connection points on the two platforms and the variable parameter is the length of links between the platforms. The kinematic model can be completely defined by providing the information about both types of kinematic parameters of the links or cables. The kinematic and the geometrical modeling of the proposed robot are described in the following sections. 


\subsection{Robot Kinematic Modeling}

The kinematic model describes the spatial motion of the end effector in time domain, about a fixed reference frame (or fixed platform). The kinematic study includes two types of models, namely the Forward Kinematic (FK) model and the Inverse Kinematic (IK) model. The forward kinematic model provides position and orientation of the end effector as a function of variable and constant kinematic parameters of all the links. Similarly the inverse kinematic model helps to find the set of joint parameters that would bring the end effector in a desired location in the workspace. The desired task of the end effector is specified in terms of its position and orientation in the workspace. The joint variables to accomplish this task are found using IK analysis. Joint variables, in turn are used to find the instantaneous coordinates of the end effector using FK analysis.

It has been well established (Innocenti and Parenti, 1990) that for parallel robots the IK solution exists in close form but the FK solution is not possible. While doing a FK analysis for parallel robots one ends up with a set of highly coupled nonlinear equations for which unique solution is not possible. Both types of kinematic models are normally required to study manipulator differential motion, its statics and to implement a desired control scheme for the end effector. Kinematic analysis is also essential to estimate the feasible workspace of the robot and to perform singularity analysis. A brief discussion on the kinematic modelling of the proposed robot is presented in the following section.

\subsection{Inverse Kinematic Analysis}

The inverse kinematics of our proposed cable driven robot is relatively simple and provides a unique solution of cable lengths for given end effector pose. In the following discussion the wire lengths have been determined in terms of the pose of the moving platform.

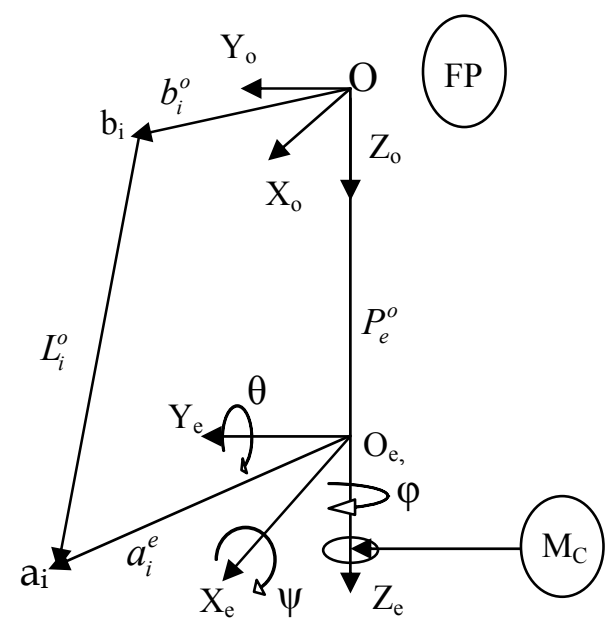

Fig. 2. A sketch of cable and position vectors of connection points on FP and MP 
The diagram presented in Figure 2 shows the position vectors of the cables in the proposed SPM. The connection points on MP and FP are denoted by $a_{i}^{\prime}$ s and $b_{i}^{\prime}$ s respectively. The connection points on the fixed platform are all in the same plane $\left(Z_{O}=0\right)$ and are placed at a radial distance ' $r_{b}$ ' from the coordinate system which is located at $O$. The position vectors $\left(b_{i}^{o}\right)$ of point $b_{i}^{\prime}$ s on the FP are defined by

$$
b_{i}^{o}=\left[\begin{array}{c}
r_{b} \cos \beta_{i} \\
r_{b} \sin \beta_{i} \\
0
\end{array}\right]
$$

where, $r_{b}$ is the radial distance from the base coordinate frame $O$. The variable $\beta_{i}$ denotes the angular position of point $b_{i}$ on FP with respect to their respective axes. The moving platform similarly has a set of connection points located on the circumference of a circle of radius $r_{a}$ and the coordinate frame attached to $O_{e}$ is about $60 \mathrm{~mm}$ above the center of mass $\left(\mathrm{M}_{\mathrm{C}}\right)$ of the MP (with reference to the position of ankle joint which is approximately $60 \mathrm{~mm}$ above moving plate level). The position vectors $\left(a_{i}^{e}\right)$ of the four connection points on the moving platform can be given as follows:

$$
a_{i}^{e}=\left[\begin{array}{c}
r_{a} \cos \alpha_{i} \\
r_{a} \sin \alpha_{i} \\
-60
\end{array}\right]
$$

The variable $\alpha_{i}$ is the angular position of point $a_{i}$ on the MP with respect to their respective axes. The position vectors of the cable lengths in terms of end poses can be expressed as a system of four equations described below:

$$
L_{i}^{o}=P_{e}^{o}+R_{e}^{o} a_{i}^{e}-b_{i}^{o} \quad i=1, \ldots 4 .
$$

where $P_{e}^{o}$ represents the position vector of point $O_{e}$ with respect to $O . R_{e}^{o}$ is the rotational transformation matrix of MP with respect to FP using a fixed axis rotation sequence of $\psi, \theta$ and $\phi$ about $X_{o}, Y_{o}$ and $Z_{o}$ axes, respectively, and can be written as below.

$$
R_{e}^{o}=\left[\begin{array}{ccc}
\cos \phi \cos \theta & -\sin \phi \cos \psi+\cos \phi \sin \theta \sin \psi & \sin \phi \sin \psi+\cos \phi \sin \theta \cos \psi \\
\sin \phi \cos \theta & \cos \theta \cos \psi+\sin \phi \sin \theta \sin \psi & -\cos \phi \sin \psi+\sin \phi \sin \theta \cos \psi \\
-\sin \theta & \cos \theta \sin \psi & \cos \theta \cos \psi
\end{array}\right]
$$




\subsection{Forward Kinematics}

Forward kinematic (FK) mapping for parallel manipulators is difficult compared to serial manipulators as it involves highly coupled nonlinear equations and their closed-form solution is not possible. However it is important to use forward kinematic solution since it is a key element in closed loop position and force control of parallel manipulators. It is also an essential block in the trajectory control of a manipulator.

Quite a few approaches are found in the literature to solve FK of parallel manipulators and a few representative works have been studied during present work. In one of the earlier works (Innocenti and Parenti, 1990) the closed form solution of FK has been simplified by merging the connection points at the two platforms which reduces the degree of polynomial representing FK. Generally in practical control and other applications a unique solution of FK is required and various numerical approaches (Deshmukh and Michael 1990) to solve non-linear equations can be used for this purpose. Several researchers have been able to linearize few of the non linear equations obtained from the kinematic analysis or have been able to reduce the degree of the set of polynomial equations (Nam and Park 2004). The system of equations of reduced order has further been solved using one of the numerical methods. Neural networks (NN), genetic algorithms (GA) and their variants have also been used by researchers to solve FK problem. Using inverse kinematics (IK) solution, one can create a database of end effector orientations and corresponding joint variables. This database can be used to train a NN and a weight matrix can be obtained for further predictions. A cascaded CMAC (Cerebella Model Arithmetic Computer) based NN to solve FK problem has been proposed by (Geng and Haynes 1991) and they have stated that the proposed algorithm is faster and more precise compared to popular back propagation algorithm. A floating point GA (Genetic Algorithm) using IK analysis has been proposed by (Boudreau and Turkkan 1996) to solve FK problem, formulating it as an optimization problem. A simple feed forward network has been used by (Yee and Lim 1997) and an accuracy of $0.017^{0}$ and $0.017 \mathrm{~mm}$, in predicting end effector pose, has been achieved. NN tuned FK model has also been used by (Oyama et al. 2002) in visually guided hand position control. A comparison of NN with various numerical methods to solve FK has been provided by (Sadjadian and Taghirad 2005). BPNN (Back Propagation Neural Network) has been used by (Li, Zhu and $\mathrm{Xu}$ 2007) where they have employed PSO (Particle Swarm Optimization) to further train NN to achieve accuracy of the order of 0.001 degrees.

\subsection{Geometric Modeling}

The kinematic models establish the correlation between the joint displacements and the position and orientation of end effector of a robot. This correlation can only be used for the static control of manipulator in the workspace. For our proposed robot the final desired angular pose of the manipulator is important and at the same time the angular velocity by which it has traversed to reach to the final location is also equally important. Thus it is essential to obtain a mapping between joint velocities and end effector velocity. This mapping can be defined by a matrix, which is called the robot Jacobian matrix. The Jacobian matrix depends on robot configuration and linearly maps the Cartesian velocity in to joint velocities. It is interesting to note that the Jacobian matrix defined for the parallel robots corresponds to the inverse Jacobian of the serial robots. To determine the Jacobian matrix of parallel robots two approaches, namely geometric approach and analytical approach can be used. In the present chapter we have used a geometric approach as discussed below. 
To determine geometric Jacobian matrix using robot geometry, initially a relation between cable lengths and end effector pose is formulated. For the subject robot this relation is given by Eq. (3). The magnitude of the cable lengths can be calculated for a given set of end effector orientation. The magnitude of each $L_{i}^{o}$ vector can be given by $l_{i}^{o}$ as shown below.

$$
\left(l_{\mathrm{i}}^{\mathrm{o}}\right)^{2}=\left(L_{\mathrm{i}}^{\mathrm{o}}\right)^{T} L_{\mathrm{i}}^{\mathrm{o}} \text {. }
$$

Taking the time derivative of the above kinematic constraint equations we obtain the following equation.

$$
2 l_{\mathrm{i}}^{o} \dot{l}_{\mathrm{i}}^{\mathrm{o}}=\left(\dot{P}_{e}^{o}+\dot{R}_{e}^{o} a_{i}^{e}\right)^{T}\left(P_{e}^{o}+R_{e}^{o} a_{i}^{e}-b_{i}^{o}\right)+\left(P_{e}^{o}+R_{e}^{o} a_{i}^{e}-b_{i}^{o}\right)^{T}\left(\dot{P}_{e}^{o}+\dot{R}_{e}^{o} a_{i}^{e}\right) .
$$

Using linear algebra identity, if ' $\mathrm{c}$ ' and ' $d$ ' are column vectors, following holds true,

$$
\mathrm{c}^{\mathrm{T}} d+d^{T} c=2 c^{T} d=2 c d^{T} .
$$

This further implies that the Eq. 6 can be rewritten as below.

$$
2 l_{\mathrm{i}}^{\circ} \dot{l}_{\mathrm{i}}^{\mathrm{o}}=2\left(P_{e}^{o}+R_{e}^{o} a_{i}^{e}-b_{i}^{o}\right)^{T}\left(\dot{P}_{e}^{o}+\dot{R}_{e}^{o} a_{i}^{e}\right) .
$$

Since the end effector is constrained to only rotational motion and the spacing between the platforms remains constant, the time derivative of $P_{e}^{o}$ should be zero. Setting $\dot{P}_{e}^{o}=0$ and further simplifying Eq. 8, we get,

$$
l_{\mathrm{i}}^{\mathrm{o}} \dot{l}_{\mathrm{i}}^{\mathrm{o}}=\left(L_{i}^{o}\right)^{T}\left(\dot{R}_{e}^{o} a_{i}^{e}\right)
$$

but since

$$
\begin{gathered}
\dot{R}_{e}^{o}=\omega_{e}^{o} \times R_{e}^{o} \\
l_{\mathrm{i}}^{\mathrm{o}} \dot{l}_{\mathrm{i}}^{\mathrm{o}}=\left(L_{i}^{o}\right)^{T}\left(\omega_{e}^{o} \times R_{e}^{o} a_{i}^{e}\right)
\end{gathered}
$$

where $\omega_{e}^{o}$ is the angular velocity vector of the end platform with respect to the coordinate system of the fixed platform. Further since $a_{i}^{e}$ and $a_{i}^{o}$ are related as shown in Eq. 12, Eq. 11 can be rewritten as Eq. 13. Rearranging the variables, Eq. 13 can be presented as Eqs. 14 \& 15.

$$
\begin{gathered}
a_{i}^{o}=R_{e}^{o} a_{i}^{e} \\
l_{\mathrm{i}}^{\circ} l_{\mathrm{i}}^{\circ}=\left(L_{i}^{o}\right)^{T}\left(\omega_{e}^{o} a_{i}^{o}\right) \\
l_{\mathrm{i}}^{\circ} l_{\mathrm{i}}^{\circ}=\left(L_{i}^{o}\right)^{T}\left(\omega_{e}^{o} \times a_{i}^{o}\right) \\
l_{\mathrm{i}}^{\circ} l_{\mathrm{i}}^{\circ}=\left(a_{i}^{o} \times L_{i}^{o}\right)^{T}\left(\omega_{e}^{o}\right)
\end{gathered}
$$

If $\dot{q}$ is the vector of link velocities and ' $t$ ' is the twist vector of the end platform, the Jacobian matrix $J(q)$ of the robot can be defined as,

$$
\dot{q}=J(q) t
$$

where ' $t$ ' is a vector of angular velocities of the end platform and is given by following. 


$$
t=\left[\begin{array}{c}
0 \\
\omega_{e}^{o}
\end{array}\right]
$$

Eq. 15 can be rearranged and compared with following matrix equation (Eq. 18) to find the Jacobian matrix as given by Equations 16 and 19 .

$$
\begin{gathered}
X \dot{q}=Y t \\
J=X^{-1} \times Y .
\end{gathered}
$$

Finally the Jacobian matrix of the proposed cable based robot can be written such that its $i^{\text {th }}$ row is given by following equation.

$$
J_{i}=\left(a_{i}^{o} \times \frac{L_{i}^{o}}{l_{i}^{o}}\right)^{T} \quad \text { where } i=1,2, \ldots ., 4 .
$$

The robot Jacobian matrix is an important parameter and is extensively used for the kinematic and dynamic analysis of the robot. This matrix shall be further used in the workspace analysis and in the actuator force analysis in the following sections.

\subsection{Workspace Analysis}

The workspace of the proposed cable based robot is difficult to analyze for the two major reasons. Firstly, the translational and orientation workspaces are achieved through coupled motion of its links or cables and the both kinds of workspaces can not be evaluated independently. Hence the workspace is defined simply as the space where the inverse and forward kinematic solutions exist. Secondly, for cable based robots (Pusey, 2003) or SPM's, their workspace is the space where sets of positive cable tensions also exist. In SPM's positive cable tensions are needed to constrain the moving platform all the time regardless of any external wrench. In other words, a manipulator pose belongs to the feasible workspace if there is at least one set of positive cable tensions forming a force closure.

Thus in SPM's, it is not only necessary to solve the closure equations but it is also essential to verify that equilibrium can be achieved with non-negative actuator (cable) forces. Workspace analysis of the SPM's is interesting due to the fact that it is constrained by more than one requirement and thus has attracted many researchers. (Stump and Kumar 2006) have approached the problem of evaluating the reachable workspace for a cable-driven parallel platform by using the tools of semidefinite programming (convex optimization) to obtain closed-form expressions for the boundaries of this workspace. Similarly in (Pusey et al. 2003), the design and workspace of a 6-6 cable-suspended parallel robot has been discussed and workspace volume is characterized as the set of points where the centroid of the moving platform can reach with tensions in all suspension cables at a constant orientation. The main contribution of (Pusey et al. 2003) is in establishing that for any geometry of platforms the largest workspace volume occurs when the moving platform (MP) is the same size as the base platform (BP). The proposed device basically has four actuated links and accommodates patient's ankle joint which acts as a central strut in the parallel device. The air muscles which are the actuating links are all in their half contracted positions initially to facilitate the antagonistic actuation of moving platform. The cables connecting both platforms are given some Pre-tension (in present study it is assumed to be 
$50 \mathrm{~N})$, by adjusting the cable lengths. Force experienced by the central strut due to this pretension in the cables is called spine force and is denoted by $F_{S}$ here. This helps in keeping all the cables tensionable in the workspace. The static force and moment balance on the MP are given as below.

$$
\begin{gathered}
F_{S}+\sum_{i=1}^{4} U_{i}=0 \\
\sum_{i=1}^{4}\left({ }^{o} m_{i} \times U_{i}\right)+\sum M_{e x t}=0
\end{gathered}
$$

Though there are no external moments applied to the MP, due to ankle stiffness, finite moments are required to move ankle joint passively. To realize the ROM exercises air muscles works antagonistically and applies the required moments at the end effector. To find corresponding tensions in individual cable the dual relationship between kinematics and statics can be used as follows:

$$
M_{e x t}=J^{T} U
$$

where ' $U$ ' is the vector of cable forces, $J$ is the geometric Jacobian matrix of the robot and $M_{\text {ext }}$ is a 3 dimensional vector containing the required moments given by Eq. (24). The rows in Eq. (24) represent the moments required to orient ankle joint about the $X_{o}, Y_{o}$ and $Z_{o}$ axes, respectively.

$$
M_{\text {ext }}=\left[\begin{array}{lll}
M_{x_{o}} & M_{y_{o}} & M_{z_{o}}
\end{array}\right]^{T}
$$

Now, to obtain the equations for the force in each of the cable, Eq. (23) is rearranged in the following manner.

where we denote

$$
\begin{aligned}
& U=\bar{J} M_{e x t} \\
& \bar{J}=\left(J^{T}\right)^{-1} .
\end{aligned}
$$

Next, at each point within the possible workspace, the equation describing the force in each cable is used to see if tension is obtainable. The actuators have a limited stroke length and hence workspace points which are not reachable by the actuators are not considered. A Matlab program is written to search the entire workspace and check for the condition of tensionability of cables and link length constraint.

The proposed robot is redundantly actuated, i.e. to achieve three degrees of freedom four actuators are used. Further since $J^{T}$ is a $3 \times 4$ matrix, its null space solution must exist and it should have one degree of freedom when $J$ is full rank. The pretension in the cables takes care that all the cables remain under tension at all times. The resulting positive actuator forces for the flexion trajectory have been plotted in Figure 3 against different sets of manipulator poses. Apparently the four actuator forces such as T1, T2, T3 and T4 are all positive during the flexion trajectory. Similarly the actuator forces for other trajectories are also evaluated. 


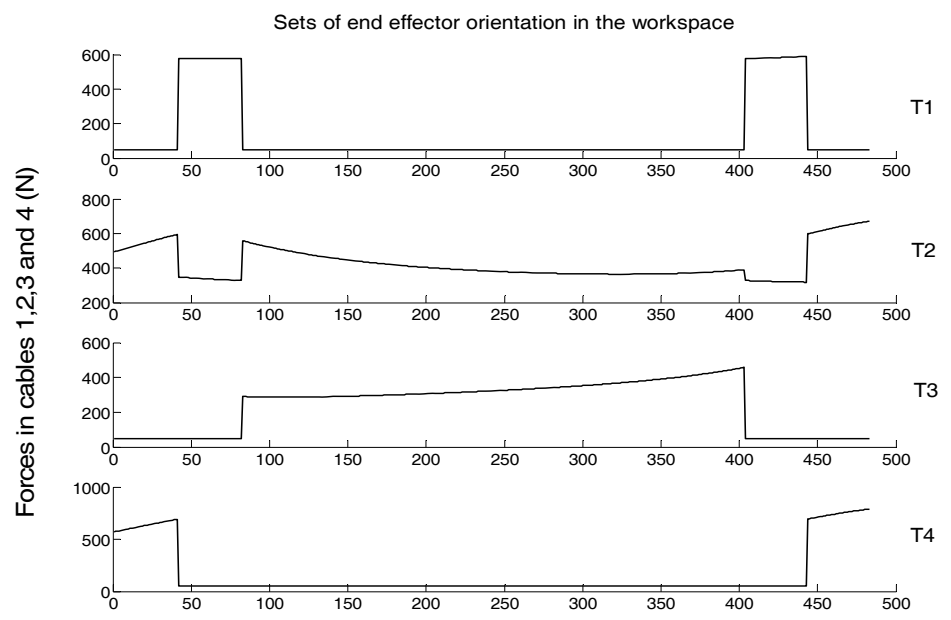

Fig. 3. Static cable forces at different end effector orientations

\section{Design Criteria of the Ankle Rehabilitation Robot}

In light of the unidirectional nature of cable forces, design of the proposed cable-based manipulator is more complicated than the rigid-link parallel robots. There are certain criteria, specific to the cable based parallel robots which require more attention. These design criteria are discussed below:

1. Maximum workspace criterion

2. Near unity condition number criterion

3. Singular value based criterion

4. Minimum force norm based criterion

5. Other criteria's

An explanation of these measures is important to state their significance.

\subsection{Workspace Criterion}

Workspace is a vital parameter in the domain of kinematic analysis and workspace analysis of the proposed robot is discussed in section 4.7. The feasible workspace volume depends on the geometrical configuration of the robot such as the size of the platforms and placement of connection points on them apart from other constraints discussed in the previous section. Thus by changing the geometrical parameters it is possible to change the volume of the feasible workspace. It is desired that the workspace of a robot under given constraints should be as large as possible for greater maneuverability. Furthermore, unlike serial robots, workspace of parallel robots is unevenly shaped (Stump and Kumar, 2006) due to their complex kinematics. This further contributes in lowering the size of the feasible workspace. Apart from the size, the quality of the workspace is also important and it is desired that the 
workspace should be free from singularities. In our study the feasible workspace is represented by an index as given below.

$$
I=\frac{\varphi_{f}}{\varphi_{T}}
$$

Here $\varphi_{f}$ is the number of workspace points (sets of manipulator orientation) which are reachable with the restricted stroke length of the actuators, and $\varphi_{T}$ is the total orientation workspace of the manipulator. As mentioned before, the manipulator orientations about $\mathrm{X}_{\mathrm{o}}$ $\mathrm{Y}_{\mathrm{o}}$ and $\mathrm{Z}_{\mathrm{o}}$ axes are given by $\psi, \theta$ and $\phi$ respectively. The limiting values of these orientations are taken as $\psi_{-30}^{+40}, \theta_{-10}^{+25}$ and $\phi_{-20}^{+20}$, considering a step size of $2^{\circ}$, the total orientation workspace given by Eq. 28, has 49000 points to evaluate.

$$
\varphi_{T}=\left(\psi_{\max }-\psi_{\min }\right)\left(\theta_{\max }-\theta_{\min }\right)\left(\phi_{\max }-\phi_{\min }\right) .
$$

In the proposed work, the robot design parameters have been optimized to achieve maximum permissible workspace volume.

\subsection{Condition Number Criterion}

As discussed in the previous sections, the Jacobian matrix ' $J$ ' of a robot maps joint rates to the Cartesian velocities of the manipulator. The condition number of this matrix is a measure of its sensitivity to changes in the kinematic variables of the robot. A robot design with near unity condition number is desirable (Khatami and Sassani, 2002) since it minimizes the error in the end effector wrench due to input errors in joint torques. The condition number can also be used to evaluate the workspace singularities. This number also reveals as how far a robot is from its present configuration to the nearest singular configuration. Stiffness of the end effector due to joint stiffnesses can also be obtained using condition number. Thus it is evident from the above discussion that the condition number is a vital design parameter and the robot configuration should be optimally designed to produce a minimum condition number close to unity. The condition number ' $k$ ' is defined as the ratio of the largest singular value ' $\sigma_{l}$ ' to the smallest singular value ' $\sigma_{s}$ ' of the matrix ' $J$ ' for a fixed orientation of the manipulator. The singular value can be further defined as the square root of the eigenvalues of $\overline{J J}^{T}$ and $\bar{J}^{T} \bar{J}$. The range of condition number is described as below.

$$
1 \leq k<\infty
$$

When the condition number approaches unity, the matrix ' $J$ ' is said to be well conditioned or far from singularities. On the contrary, if the condition number is higher, the matrix is said to be ill conditioned. An ill conditioned Jacobian matrix will further magnify the kinematic or dynamic error present in the robot motion. Sometimes to avoid an infinite right hand side bound, an inverted form of the condition number referred to as the conditioning index (C.I. ) can also be used. 


$$
C . I .=\left(\frac{1}{k}\right)
$$

where $0 \leq C . I . \leq 1$

The condition number is generally obtained at each individual point in the feasible workspace region, for a fixed orientation of the end effector. Though condition numbers at individual manipulator orientations are useful information, the Global Conditioning Number (GCN) (Khatami and Sassani, 2002) is normally used to analyze the behavior of the condition number over the entire workspace volume. In the present work robot performance has been defined by Global Condition Number (GCN) as given by,

$$
G C N=\frac{\int_{W}(k) d w}{\int_{W} d w}
$$

where ' $k$ ' is the condition number at a given orientation and ' $W$ ' is the feasible workspace. Since it is difficult to calculate the exact solution to the integrals mentioned above, GCN is discretely defined and expressed as below.

$$
G C N=\frac{\sum_{i=1}^{n}(k)}{n}
$$

Here $n$ is the total number of discrete feasible points constituting the workspace and the numerator is the sum of condition numbers obtained at different points in the feasible workspace volume grid. The GCN is bounded by the range as given.

$$
1 \leq G C N \leq \infty
$$

Here, when the GCN is a large number the entire workspace tends to be ill conditioned and when the GCN is near one the entire workspace is said to be well conditioned. GCN further depends on the robot configuration which is defined by arrangements of connection points at both the platforms and the link lengths. Hence there exists an optimum robot configuration for a good GCN and performance thereof. To ensure that all the points in the workspace provides a condition number within certain range, the maximum value of the condition number for a particular robot design over the entire workspace can be obtained and minimized. Once the maximum GCN is minimized, it can be ensured that

1. The final GCN represents the average behavior of condition number over the feasible workspace.

2. The condition number all over the feasible workspace is always less than the minimum GCN value.

\subsection{Singular Value Based Criterion}

Singular values are important measure of kinematic behavior of the robot and provides an assessment on its controllability. The manipulator loses or gains extra degrees of freedom 
when it enters in to a singular configuration. A kinematic singularity occurs when the determinant of the Jacobian matrix becomes zero or loses rank at a particular configuration in the workspace.

$$
\operatorname{det}(J)=0
$$

Referring to Eq. (20), it is apparent that when ' $J$ ' is singular and its null space is non-zero, there will be certain non-zero Cartesian vectors $(t)$ resulting in zero joint vectors $(\dot{q})$. This further means that, despite the joints being locked, the end effector can still have some infinitesimal motion in a particular direction and gains one or more degrees of freedom. Hence it is essential to optimize the design parameters of the robot, in order to minimize the number of singular points in the workspace. The condition number provides a fraction of maximum and minimum singular values. Thus a criteria based on minimization of maximum singular values shall yield a workspace free from singularities. However in the present optimization problem when GCN is minimized (which is the ratio of maximum singular value to the minimum singular value), the maximum singular value also get minimized.

\subsection{Minimum Actuator Force Based Criteria}

Since we intend to design a wearable robot for ankle joint rehabilitation treatment, it is desired to keep the length of the robotic device similar to the length of the patients' leg. The length of the robot is governed by the length of its actuators hence the actuators should be kept as small as possible. Further the size of these actuators depends on the cable forces calculated (Eq. 25) in Section 4.7. Longer air muscles are required for higher forces in the individual links. The lengths of the actuators can be minimized by lowering the actuator force requirements. Higher actuator forces may cause the cables to break and these forces may also produce undesired cable elongation affecting the positional accuracy adversely. Once again it is apparent that the actuator forces are the function of robot's geometrical parameters. By selecting connection points farther from the axis of rotation, the forces can be greatly reduced. To minimize the actuators force vector it is convenient to summarize the set values of force vector as a single number. Vector norms are generally used to represent the vector in a single value. Three types of vector norms are generally used such as, 1-norm, 2norm or $\infty$-norm. 2-norm or Euclidean length is preferred (Hassan and Khajepour, 2008) over the other two norms because it is more sensitive towards changes in larger force components. 1-norm is equally sensitive to all the force components and $\infty$-norm is only sensitive to the changes in the largest force component. In the present study, 2-norm or the Euclidean distance of the actuator forces has been considered and can be given as below:

$$
\|U\|_{2}=\|0-U\|
$$

\subsection{Miscellaneous Criteria}

There are other design criteria which are specific to the application of the robot. Such criteria are size of the manipulator and the fixed platform, range of motions of the robot, material selection based on strength criteria, etc. 
Despite the advantages discussed in previous sections the parallel manipulator has some innate problems owing to its closed chain mechanism. A parallel robot basically has several serial robots connected in parallel and its feasible workspace is the intersection of the individual workspace contribution of its joints. Hence the feasible workspace of our robots is small in size. Apart from the size and the shape, the workspace is also affected by the kinematic singularities. The proposed parallel robots due to its complex mechanism have three kinds of singularities such as inverse kinematic singularity, direct kinematic singularity and the combined singularities.

The design of cable based parallel robot is again more challenging since it has an added constraint on the workspace in the form of tensionability. The feasible workspace of this robot is the euclidean space where the robot manipulator can reach with positive tension in all its cables. Thus apart from the singularities the condition of tensionability is also required to be checked to evaluate the feasible workspace for the manipulator.

The proposed robot is to be used for ankle joint rehabilitation treatments and the subjects are supposed to fix their foot and the ankle into the moving platform. It is evident that the device has to be robust to take care of the physical differences in the shape and size of different patient's foot and ankle. Differences in the foot of different subjects also amount to the small variations in the robot kinematic and dynamic parameters. Furthermore, when in actual use, the effective location of the ankle joint in the robotic device also changes to some extent. This is due to the fact that the ankle joint is approximated by two joints (as explained in section 3.1) in series and different ankle motions are the result of a coupled rotation about these joints realized by one of these joints.

The kinematic and geometric parameter variations in the joint space results in the corresponding Cartesian parameters variations. Owing to this fact, the Jacobian matrix of the robot, which relates the joint and Cartesian rates, is required to be well conditioned. It is apparent that if the Jacobian matrix is not well conditioned then the small changes in the joint variables will result in very large changes in the Cartesian variables. This will further lead to difficulty in manipulator control and large trajectory following errors. Nevertheless, by choosing optimal geometric parameters of the robot, the condition number of the Jacobian matrix can be improved and the design can be made robust to parametric variations.

Another problem with cable based parallel robot is that the cables can not be subjected to very high forces. Higher forces in the cables may cause breakage or undesired elongation of the cable which will adversely affect the positional accuracy. Moreover to achieve higher forces, the required length of the air muscles also increases proportionally (as discussed in Section 5.4) which is not desirable. By optimizing the geometrical parameters of the robot, the force requirements can also be minimized.

It can be concluded from the above discussion that the design of the robot is required to be optimized to maximize its workspace, and minimize the condition number and the actuator force requirements. The dimensional analysis and optimization of the robot design may include the key design parameters such as the shapes and sizes of the parallel platforms, locations of the connection points of the cables on both these platforms and the lengths of cables. Such a design optimization is essential to reduce the above mentioned shortcoming of the robot while maintaining its inherent merits. 


\section{Design Optimization}

Though parallel robots are in use in a wide range of applications, their potential has not been completely exploited. The reasons are obvious and have been discussed in the previous section. To address the issues of the workspace, singularity and the robust design of parallel robots, trial and error approach is normally used. This approach is based on rigorous experiments or simulation runs and intuitive judgments on the results thereafter. The main drawback of this approach is that with an increase in number of tunable parameters the required number of simulation runs increases exponentially. Moreover tuning of all the design parameters simultaneously is difficult and time consuming. Some of the researchers have tried to optimize one or more of the design objectives using some numerical methods. Several performance indices such as manipulability, isotropy, dexterity index, conditioning index, global conditioning index and global isotropy index have been defined by different researchers (Khatami \& Sassani, 2002) to compare the performances of competing manipulator designs.

In a recent study (Hassan \& Khajepour, 2008) the actuator forces in a cable based parallel manipulator have been optimized using a minimum norm solution. Authors have used Dykstra's projection method to optimally distribute the actuator forces among the cables and the redundant limbs. Though the force distribution among links has successfully been optimized to provide minimum norm solution, the geometrical parameters have not been tuned to minimize the actuator forces. Genetic algorithms (GA) have been used by (Sergiu et al., 2006) to optimize the workspace of a 2-DoF parallel robot using a mono-objective function. A novel kinematic design method has been implemented by (Liu, 2006) and various performance indices such as global conditioning workspace, global conditioning index and global stiffness index have been used to obtain the design parameters. A multicriteria optimization based on conventional weighted average approach has been performed by (Lemay \& Notash, 2003). The authors have proposed a combination of GA and simulated annealing algorithms to optimize workspace, dexterity and mass \& size of the manipulator simultaneously. Workspace and stiffness of a modular parallel robot have been studied and optimized by (Merlet, 2003). The author has proposed a branch and prune type algorithm to improve the robot performance. A new performance index called space utilization has been proposed by (Stock \& Miller, 2003) to evaluate the optimal kinematic design of a linear Delta robot. They have used an exhaustive search minimization method to optimize mobility, workspace and manipulability. Global conditioning index has been optimized as a result of altering the length of links by (Khatami \& Sassani, 2002). The authors have used a nested implementation of two GA to obtain a mini-max genetic solution. GA has been used with constraints defined as penalty functions by (Su et al., 2001) to minimize the minimum condition numbers in the entire workspace. To validate and verify the algorithm, results obtained from GA have further been compared with the Quasi-Newton method. Architectural optimization of a 3-dof parallel robot has been performed by (Tsai \& Joshi, 2000) to maximize the global conditioning index.

\section{Mathematical Formulation}

There are three major issues to be addressed in the proposed design. For better controllability the robot should have the condition number value close to unity. Thus the Global condition number is required to be minimized. The workspace of parallel robots is a 
critical parameter and it is required to be maximized. Lastly for a good design the norm of the actuators force vector is required to be minimized.
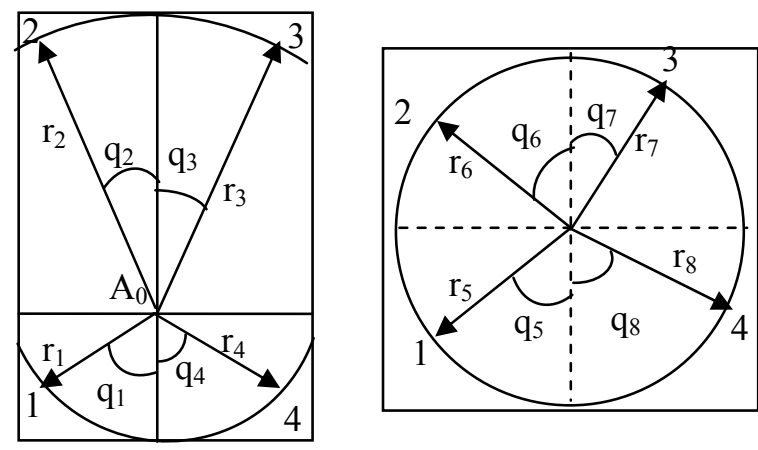

Fig. 4. Rectangular end platform and circular fixed platform with their respective geometrical parameters

Thus we have three objectives to optimize and out of these two are required to be minimized and one objective function is required to be maximized. Since the robot's performance is very sensitive (Merlet, 2003) to its geometry, we have identified sixteen geometrical variables and have varied them to obtain different robot configurations. These variables are the polar coordinates of eight connection points on both the platforms. The formulation of the optimization problem is discussed in the following section.

\subsection{The Objective Functions}

(1) Minimize Global Condition Number (GCN):

$$
\begin{aligned}
& f\left(q_{p}, r_{p}\right)=G C N(i)+P(i, j) \\
& G C N \quad(i)=\frac{\int_{W}(k) d W}{\int_{W} d W}
\end{aligned}
$$

Here, the condition number of a particular robot design at a given orientation is denoted by ' $k$ ' and ' $W$ ' is the feasible workspace explained in the previous section 4.7. The eight sets of polar coordinates $\left(q_{p}, r_{p}\right)$ consisting of angular positions and radial distances are used to specify the location of eight connection points (Figure 4) on the two platforms, ' $i$ ' is the number of robot designs and $j \in W$. Further $P(i, j)$ is a penalty term defined as below.

$$
P(i, j)=\left(\frac{\sigma_{\text {max }}(J(i, j))}{\sigma_{\text {min }}(J(i, j))}\right)_{\text {max }}
$$


Where $\sigma_{\min }$ the smallest singular value and $\sigma_{\max }$ is defined as largest singular value of the robot's jacobian matrix $J$ for each workspace point.

(2) Maximize the workspace utilization index (I)

$$
I=\frac{\varphi_{f}}{\varphi_{T}}
$$

Here $\varphi_{f}$ represents the number of workspace points which are reachable with the restricted stroke length of the actuator, and $\varphi_{T}$ is defined as below.

$$
\varphi_{T}=\left(\psi_{\max }-\psi_{\min }\right)\left(\theta_{\max }-\theta_{\min }\right)\left(\phi_{\max }-\phi_{\min }\right)
$$

Here $\psi, \theta$ and $\phi$ are roll, pitch and yaw angles for the moving platform.

(3) Minimize Tension norm in the workspace

$$
\|U\|=\left\|\bar{J} M_{\text {ext }}\right\|
$$

Where

$$
\bar{J}=\left(J^{T}\right)^{-1}
$$

And

$$
M_{e x t}=\left[\begin{array}{lll}
M_{x_{0}} & M_{y_{0}} & M_{z_{0}}
\end{array}\right]^{T}
$$

and ' $U$ ' is the vector of cable forces present in each cable, $J$ is the Geometric Jacobian matrix of the robot and $M_{e x t}$ is a 3 dimensional vector containing the required moments to move ankle joint.

\subsection{The Kinematic Constraints}

(1) Workspace Constraints: To find the optimum location of the connection points on the platforms their polar coordinates are varied to search the complete feasible area of the platforms. It is apparent that by changing the polar coordinates, the whole platform area can be investigated. However the points are varied within certain limits (Equations 43 \& 44) so that the patient can comfortably place his foot in the robot. Variables mentioned in the Eq. 43 have been shown in Figure 4. Here $\gamma_{1}$ and $\gamma_{2}$ are two convenient limits (70 mm and 140 $\mathrm{mm}$ ) describing the variation in the size of both platforms.

$$
\begin{aligned}
& \frac{\pi}{6} \leq q_{1}, q_{4} \leq \frac{\pi}{2}, \frac{\pi}{4} \leq q_{2}, q_{3} \leq \frac{\pi}{2} \\
& \text { and } \frac{\pi}{4} \leq q_{5}, \ldots, q_{8} \leq \frac{\pi}{2} \\
& \gamma_{1} \leq r_{1}, \ldots ., r_{8} \geq \gamma_{2}
\end{aligned}
$$


(2) Stroke length check for $m^{\text {th }}$ link: Since the air muscle actuator has a limited stroke length, it is essential to ascertain that for all different orientations of end effector the change in the link length or the stroke length is always within permissible limits.

$$
l_{m}^{\min } \leq l_{m} \leq l_{m}^{\max }
$$

(3) Tensionability Constraints: To ensure that all the cables remain in tension for all the required orientations of end effector while carrying out ROM exercises. $U_{m}$ is the value of tension in the $m^{\text {th }}$ cable.

$$
U_{m} \geq 0
$$

(4) Checking maximum tension in a link: Once again, the air muscle actuators being used have a limited force capacity $\left(\beta_{1}=700 \mathrm{~N}\right)$ and hence it is required to check that the maximum tension $\left(\left[U_{m}\right]_{\max }\right)$ in a link should not exceed this value.

$$
\left[U_{m}\right]_{\max } \leq \beta_{1}
$$

This is a multi-objective and multivariable constrained optimization problem. To solve such problem there are two main approaches mentioned in the literature (Deb, 2005) such as, the weighted formula approach and the Pareto optimal solution approach. To carry out the optimization, conventionally, direct search or gradient decent methods are used but these techniques become less efficient when the search space is large and is finely discretized. Further these methods sometimes get trapped into local minima and fail to provide a global optimum solution. It has been observed that when the objective function does not change over certain points in succession, these traditional methods become less effective. We propose to use Genetic Algorithms in the present optimization problem. GA works with population of points and processes them simultaneously and hence is more likely to give a global solution. As is evident from Figure 8-10, the values of condition number are quite similar in the neighborhood of any given point and the variation is very smooth. Thus discretizing the workspace does not lead to any information loss and this fact further supports our choice of GA.

\section{Multi-criteria Optimization Techniques}

\subsection{The Weighted-Formula Approach}

Conventionally to solve a multi-objective problem it is transformed it into a single-objective problem. This is typically done by assigning a numerical weight to each objective (evaluation criterion) and then combining the values of the weighted criteria into a single value by either adding or multiplying all the weighted criteria. That is, the value $\mathrm{V}$ of a given candidate model is typically given by one of the two kinds of formula:

$$
V=w_{1} \times f_{1}+w_{2} \times f_{2}+\ldots+w_{n} \times f_{n}
$$

Or

$$
V=f_{1}^{w_{1}} \times f_{2}^{w_{2}} \times \ldots f_{n}^{w_{n}}
$$


Here $w_{n}$ denotes the weight assigned to function $f_{n}$ and $\mathrm{n}$ is the number of evaluation criteria. A weighted formula approach has been used by (Lemay and Notash, 2003) to construct a configuration engine for robot design optimization. This approach has several associated benefits, such as, the importance of one objective over the rest can be controlled using appropriate weights and the finally obtained result is usually a Pareto optimal solution (a solution satisfying all the objectives equally). Further the approach is fairly simple and easy to use and thus is very popular. However it suffers from certain drawbacks and worse of them is the ad-hoc selection of weights for different objectives. Generally the choice of weights is based either on some trial and error experiments with different weights or on the intuitive judgment of the user. Both these choices are subjective and lack a logical ground. Further different quality measure of varying units and scales are required to add in a single objective function which is not correct mathematically. However appropriate normalizing procedures can be used to address this problem.

\subsection{The Population Based Approach}

This approach entails the use of population of Evolutionary Algorithms (EA) to expand the search without using the idea of Pareto optimality. The Vector Evaluated Genetic Algorithm (VEGA) proposed by (Schaffer, 1985) is an example of this approach. This basically is the simple GA but with different selection process adapted to perform multi-objective optimization. Subpopulations for each of the objective function are selected separately based on their individual fitness. These subpopulations later, are mixed and other genetic operators such as crossover and mutation are performed on them as usual. Hence this approach has an advantage over weighted formula method that the non-commensurable or the criteria of different units are treated separately with diverse subpopulation. However the implementation of this approach is complex compared to the previous method. Further drawback of this approach is the absence of Pareto dominance concept in the selection process. Thus a design which offers a good compromised solution for all the criteria but fails to provide best solution for one of them, get discarded. Nevertheless, for the problems having large number of objectives or when the objectives are of similar nature, this approach is useful since it creates a bias in the selection process.

\subsection{The Pareto Approach}

The basic idea of the Pareto approach is that, instead of transforming a multi-objective problem into a single-objective function, all the objective criteria are evaluated simultaneously for a population. The dominating solutions emerging from the population are selected and ranked, based on their dominance. Two design solutions $\left(\mathrm{sol}_{1}\right.$ and $\left.\mathrm{sol}_{2}\right)$ are compared and one of them (i.e. sol $_{1}$ ) is termed as dominated only if it is strictly better than the other solution (i.e. $\mathrm{sol}_{2}$ ) with regard to at least one of the objectives. Further sol $_{1}$ should not be worse than $\mathrm{sol}_{2}$ for other objectives. A number of Pareto approaches are mentioned in the literature and a comprehensive survey of these can be found in (Coello et al., 2002). In the present study a simple genetic algorithm based approach has been used to generate a Pareto optimal front. Despite their obvious advantages over other approaches, Pareto optimal solutions do suffer with the problem of subjectivity in choosing the best solution from the Pareto optimal solution set. 


\subsection{Genetic Algorithm Methodology}

The fitness function and parameter selection have been discussed here and for a detailed reading on GA, the book (Deb, 2005) is recommended.

\subsubsection{Generation}

Initial population of 20 binary coded strings of 160 bits was generated using Knuth's random number generator (Knuth, 2000). Sixteen variables $q_{1}, \ldots q_{16}$ as shown below, one for each connection point location have been defined. The binary string of 160 bits has 10 bits assigned for each of the sixteen variables, thus the solution accuracy of the order of $10^{-3}$ can be achieved.

$$
\underbrace{1101 . .1}_{q_{1}} \underbrace{1010 . .0}_{q_{2}} \underbrace{0110 . .1}_{q_{3}} \underbrace{1011 . .0}_{q_{4}} \underbrace{1010 . .1}_{q_{5}} \ldots \ldots \ldots \underbrace{1001 . .0}_{q_{16}}
$$

The binary fractions of individual variables are converted into decimal numbers and the three performance criteria are evaluated to finally calculate the fitness function. The steps involved in GA have been further explained in subsection 7.5.

Remark: In the proposed work initially 20 robot designs are generated randomly and are represented in a binary string. The benefit of using GA is that the initial population needs not to be very large because the initial population keeps evolving and changing after each iteration.

\subsubsection{Fitness Evaluation}

In order to evaluate each string in the population, its fitness must be calculated. Generally GA is used for maximization problems and hence for the present case of multi-modal and multi-criteria optimization all the objectives have been converted to maximization functions. The binary strings are decoded to real numbers and the three performance criteria are evaluated to finally calculate the fitness function.

\subsubsection{Reproduction}

The population initially selected may not have all the good strings in terms of their fitness values. Thus good strings have been analyzed based on individual fitness and their multiple copies have been selected in the mating pool. The Roulette-Wheel Selection method (Deb, 2005) has been used for reproduction.

\subsubsection{Crossover and Mutation}

A four point crossover with 0.95 probability has been used and mutation has been performed with 0.01 probability. Generally crossover probability is kept close to one so that all the parent strings may get a chance to crossover. Mutation helps in finding a global optimum solution but to avoid a random search its probability is kept low. 


\subsection{Proposed GA Algorithm}

In the proposed work efforts have been made to explore the advantages of two of the approaches discussed in subsections 7.1 \& 7.3, such as, weighted formula and the Pareto optimal approach. The steps involved in the proposed algorithm are explained below.

Step 1. Choose termination criteria (either number of epochs or the error function could be used). In the present case we have chosen an error function which is defined as ( $\varepsilon=1-$ Fitness ) and the algorithm terminates when the error function is 0.01 or the fitness is 0.99 .

Step 2. Initialize a random population of 20 binary strings with 160 bits in each binary. There are sixteen design variables in the present problem and each of them are represented by ten bits hence every binary corresponds to an individual robot design.

Step 3. Convert binary values of sixteen design variables to the decimal values taking their universe of discourse into account as shown below.

$$
x_{i}=x_{i}^{L}+\frac{x_{i}^{U}-x_{i}^{L}}{2^{l_{i}}-1} \times(\text { decoded value of binary string })
$$

Where $x^{L}$ and $x^{U}$ are the lower and the upper limits of the design variable, $l_{i}$ is the length of binary string which is 10 in the present case.

Step 4. Evaluate the three objective criteria for these 20 robot designs and normalize them. The values of the criteria are scaled in the range 0 to1 using following linear mapping. For example GCN of the ith robot design is normalized as below:

$$
G C N_{\text {normalized }}=\frac{G C N_{i}-G C N_{\min }}{G C N_{\max }-G C N_{\min }}
$$

Since GA can only maximize a function and can not minimize, care has been taken to convert all the criteria into maximization functions by inverting the function which require minimization. Three constraints for the limiting values of three criteria are defined in the algorithm and any solution outside these values is discarded. The maximum values are selected on the basis of design limitations and are as below.

$$
0.25 \leq \varphi, \quad G C N \leq 4.0 \text { and }\|U\| \leq 2000
$$

Step 5. Calculate the fitness function (Eq. 51) with equal priorities i.e. $\mathrm{w}_{1}=\mathrm{w}_{2}=\mathrm{w}_{3}=0.3333$.

$$
\text { Fitness }(i)=w_{1} \times G C N(i)+w_{2} \times\|U\|(i)+w_{3} \times \varphi(i)
$$

Step 6. Evaluate the individual fitness of the initial 20 robot designs. Using roulette wheel selection method (subsection 7.4.3) create multiple copies of binaries in the mating pool, based on their individual fitness.

Step 7. Search for any non-dominated solution as discussed in section 7.3 and store their copies in a vector.

Step 8. Perform crossover operator on randomly selected parents and later perform mutation operator on the crossed-over binaries.

Step 9. Check for the termination criteria, if not met, go to Step 3 else terminate. 
The sensitivity of the three optimization criteria has been checked by changing their weights in the fitness function. The results of sensitivity analysis from the above algorithm have been shown in Table 2 .

\section{Results and Discussions}

The weighted criteria used and the final results obtained in the present multi-criteria optimization problem are shown below in Table 2 .

\begin{tabular}{|l|l|c|c|c|}
\hline & Priorities $\left(\mathrm{w}_{\mathrm{n}}\right)$ & $\begin{array}{c}\text { Global Condition } \\
\text { Number }(\mathrm{GCN})\end{array}$ & $\begin{array}{c}\text { Actuator } \\
\text { Force } \\
\text { Norm }(\mathrm{N})\end{array}$ & $\begin{array}{c}\text { Permissible } \\
\text { Workspace } \\
\text { Fraction }(\varphi)\end{array}$ \\
\hline Case I & $\mathrm{w}_{1}=\mathrm{w}_{2}=\mathrm{w}_{3}=0.333$ & 2.497 & 1537.7 & 0.80 \\
\hline Case II & $\mathrm{w}_{1}=0.8, \mathrm{w}_{2}=0.1, \mathrm{w}_{3}=0.1$ & 2.11 & 1612.9 & 0.84 \\
\hline Case III & $\mathrm{w}_{1}=0.1, \mathrm{w}_{2}=0.8, \mathrm{w}_{3}=0.1$ & 2.225 & 1439.9 & 0.70 \\
\hline Case IV & $\mathrm{w}_{1}=0.1, \mathrm{w}_{2}=0.1, \mathrm{w}_{3}=0.8$ & 2.45 & 1657.18 & 0.95 \\
\hline
\end{tabular}

Table 2. Results from four experiments with different weighted criteria.

Further, the optimum design parameters obtained from the equal priority objectives are also shown in Table 3. Experiments with different priorities (Table 2) reveal that there is a trade off between workspace and actuator forces. When minimization of actuator forces is given more weight $\left(\mathrm{w}_{2}=0.8\right)$, the available workspace reduces to $70 \%$. Conversely when the workspace maximization is given higher weight $\left(\mathrm{w}_{3}=0.8\right)$, the actuator force norm increases to $1657.18 \mathrm{~N}$. However the global condition number seems to be less sensitive for change in priorities. A close look at the design parameters (units are degrees and millimeters) shown in Table 3 reveals that the designs are closely symmetric. Also it can be observed that when the actuator force minimization is given more weight the connection points, as expected move farther from the center of rotation. On the other hand when workspace utilization is given more priority the connection points move close to the ankle joint.

\begin{tabular}{|c|r|r|r|r|r|r|r|r|r|r|r|r|r|r|r|r|}
\hline & $\mathrm{q} 1$ & $\mathrm{q} 2$ & $\mathrm{q} 3$ & $\mathrm{q} 4$ & $\mathrm{q} 5$ & $\mathrm{q} 6$ & $\mathrm{q} 7$ & $\mathrm{q} 8$ & $\mathrm{r} 1$ & $\mathrm{r} 2$ & $\mathrm{r} 3$ & $\mathrm{r} 4$ & $\mathrm{r} 5$ & $\mathrm{r} 6$ & $\mathrm{r} 7$ & $\mathrm{r} 8$ \\
\hline $\begin{array}{c}\text { Case } \\
\text { I }\end{array}$ & 46 & 35 & 34 & 50 & 65 & 72 & 71 & 63 & 97 & 133 & 136 & 99 & 81 & 89 & 86 & 81 \\
\hline $\begin{array}{c}\text { Case } \\
\text { II }\end{array}$ & 25 & 23 & 45 & 37 & 75 & 69 & 61 & 57 & 85 & 98 & 102 & 95 & 89 & 78 & 106 & 73 \\
\hline $\begin{array}{c}\text { Case } \\
\text { III }\end{array}$ & 46 & 22 & 20 & 48 & 66 & 60 & 59 & 64 & 96 & 149 & 138 & 86 & 97 & 100 & 117 & 88 \\
\hline $\begin{array}{c}\text { Case } \\
\text { IV }\end{array}$ & 48 & 37 & 31 & 58 & 66 & 57 & 58 & 57 & 81 & 141 & 143 & 85 & 71 & 81 & 89 & 71 \\
\hline
\end{tabular}

Table 3. Designs from the equal priority objective optimization.

The design of the proposed robot is expected to be left-right symmetric because it is intended to be used for both right and left ankle joint rehabilitation treatments. Though the symmetry requirement was not considered as a constraint in the design optimization process, we finally get designs which are almost left-right symmetric and deviate moderately. 
While using the weighted formula method all the objective functions were normalized and scaled in the range, from zero to unity. Initially all the objectives were given equal priorities and the objective function comprising the three objectives was optimized using GA. The optimized design as can be seen from the Table 2, has a global condition number as 2.4972 which is close to one and is acceptable. The design so obtained is able to use $80 \%$ of the total workspace volume and requires a force norm of $1537.7 \mathrm{~N}$ to move ankle joint passively. Though the condition number and the workspace utilization are acceptable but the force norm requirement is higher and is not adequate. One possible approach to address this problem is to use a different weighted formula which gives more priority to the force norm minimization compared to the workspace utilization and the condition number. Thus to explore more possibilities we further used three different types of weighted formulas giving priorities to the three objectives respectively. The results from these three objective formulations are shown in the Table 2. The difficulty in choosing one of these designs is that while giving more priority to a particular objective, we have to compromise on part of other performance criteria. Such as, if the minimization of the force norm is given more priority we do not get good condition number and the workspace remains under-utilized. This fact is evident from the results provided in Table 2. To overcome this problem we need more number of designs, displaying better performances, to make a decision on the final design. This led us to the concept of the Pareto optimal set solutions. Using non-dominated solutions approach in the present algorithm, we were able to obtain a number of good solutions providing acceptable solutions for all the three objectives simultaneously.

We have created a vector (Step7 in Section 7.5) in our algorithm to store the designs which have dominating performance in each epoch. These designs are later analyzed and the finally dominating designs out of these are selected using the same measure of dominance as discussed in Section 7.3. A plot of dominant designs is shown in Figures 5-7 and the dominating designs with their performance criteria are listed in Table 4. Design parameters from the finally dominating solutions are also displayed in Table 5. For clarity of the presentation, the Pareto optimal surface obtained for the three performance criteria is shown discretely in three plots (Figures 5-7). It is evident from the results shown that the robot performance is sensitive to its geometry and the performance criteria are also interdependent particularly the workspace and the actuator forces.

The Pareto optimal front provides at least eleven sets of solutions to choose from. Figure 5 shows solutions 2, 4, $7 \&$ 11, providing minimum GCN and actuator forces. Design solutions $6,7 \& 10$ are shown in Figure 6, which provide maximum workspace and minimum GCN. Similarly the optimal design solutions 1, 2, 3, 5, 8, 9 \&10 offering minimum actuator forces and maximum workspace are shown in Figure 7 . Though all of these solutions are acceptable, solution sets 1,8 and 6 appears to be better solutions. However the workspace utilization for these solutions is low and is $70 \%-80 \%$ of the total workspace volume. The workspace utilization index, used in the present work is the ratio of the points satisfying actuator stroke length constraint and the total assumed workspace volume. It was found that by increasing lengths of the air muscles by $20 \%$ the workspace utilization can be increased significantly. A suitable trade-off between the performance criteria is required to establish a particular design from the available Pareto optimal solution sets. 


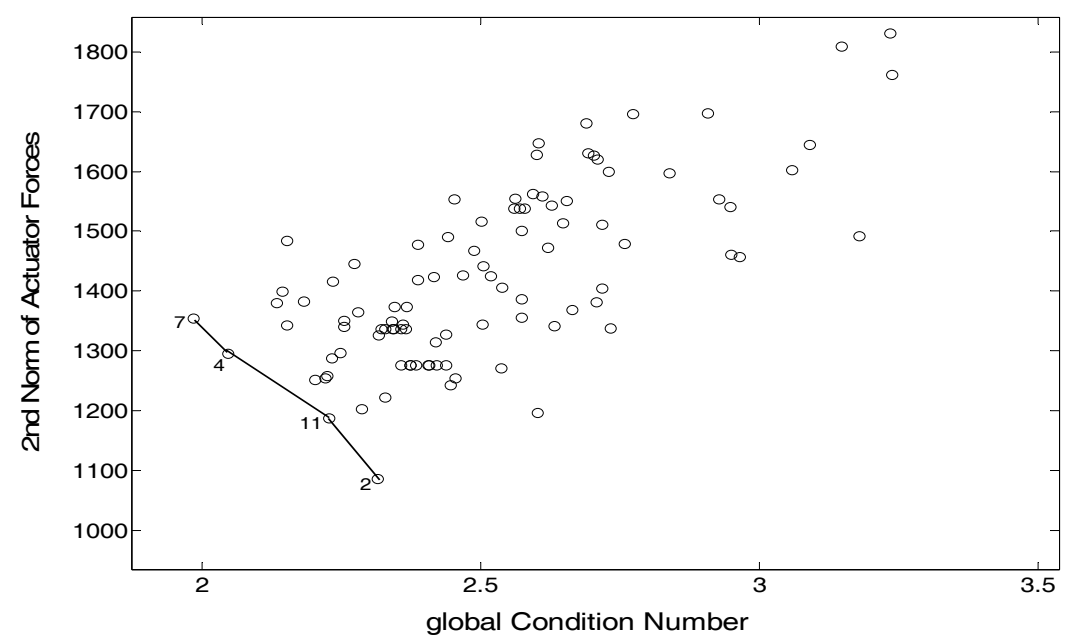

Fig. 5. Pareto optimal front for the norm of actuator forces $(\mathrm{N})$ and the global condition number

Further the quality of the available workspace can also be checked with the behavior of the condition number. Hence an exhaustive search was performed in the entire workspace of the final design (D8). The variation in the condition number was plotted against $X$ and $Y$ Euler's angle as shown in Figures 8-10. It was found that the range of condition number variation in the entire feasible workspace was from 2.3322 to 3.2241 , which is within the required limits.

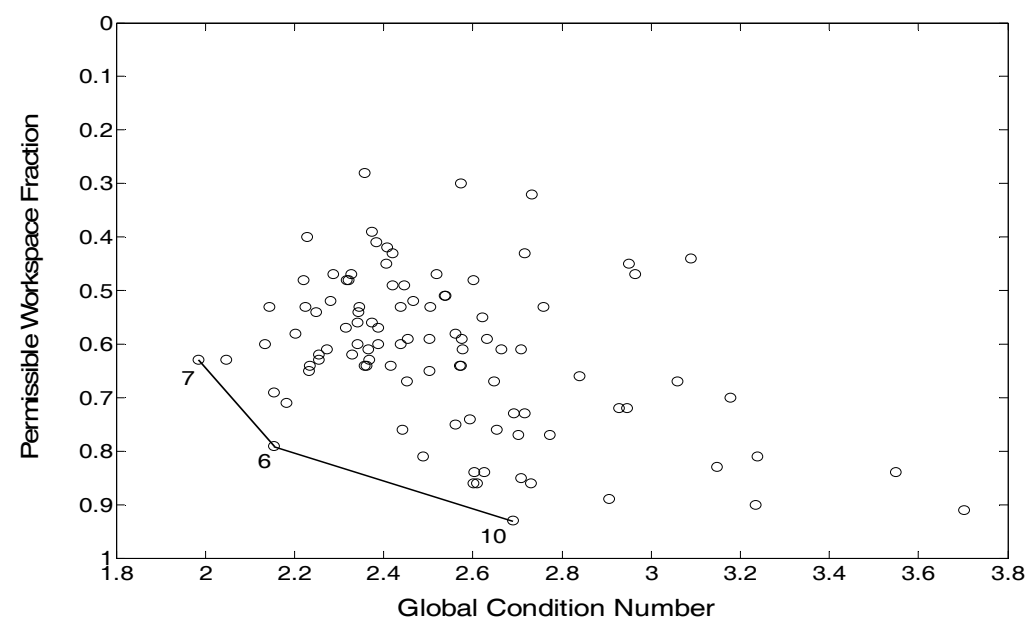

Fig. 6. Pareto optimal set for permissible workspace fraction and the norm of actuator forces $(\mathrm{N})$ 


\begin{tabular}{|c|c|c|c|}
\hline $\begin{array}{c}\text { Design } \\
\text { Index }\end{array}$ & Global Condition Number & $\begin{array}{c}\text { Actuator Force } \\
\text { Norm }(\mathrm{N})\end{array}$ & $\begin{array}{c}\text { Permissible } \\
\text { Workspace Fraction }\end{array}$ \\
\hline 1 & 2.182 & 1382.098 & 0.71 \\
\hline 2 & 2.315 & 1085.602 & 0.57 \\
\hline 3 & 2.6266 & 1543.118 & 0.84 \\
\hline 4 & 2.0468 & 1295.654 & 0.63 \\
\hline 5 & 2.1531 & 1342.863 & 0.69 \\
\hline 6 & 2.1534 & 1484.412 & 0.79 \\
\hline 7 & 1.9843 & 1353.789 & 0.63 \\
\hline 8 & 2.4875 & 1467.597 & 0.81 \\
\hline 9 & 2.2324 & 1288.175 & 0.65 \\
\hline 10 & 2.6893 & 1680.771 & 0.93 \\
\hline 11 & 2.2278 & 1186.855 & 0.4 \\
\hline
\end{tabular}

Table 4. Performance criteria for the designs of the Pareto optimal front.

\begin{tabular}{|r|r|r|r|r|r|r|r|r|r|r|r|}
\hline & \multicolumn{1}{|c|}{ D1 } & \multicolumn{1}{c|}{ D2 } & \multicolumn{1}{c|}{ D3 } & \multicolumn{1}{c|}{ D4 } & \multicolumn{1}{c|}{ D5 } & \multicolumn{1}{c|}{ D6 } & \multicolumn{1}{c|}{ D7 } & \multicolumn{1}{c|}{ D8 } & \multicolumn{1}{c|}{ D9 } & \multicolumn{1}{c|}{ D10 } & D11 \\
\hline q1 & \multicolumn{1}{|c|}{43.6} & 43.6 & 66.5 & 43.6 & 32.1 & 50.4 & 40.1 & 72.2 & 50.4 & 59.0 & 43.6 \\
\hline q2 & 28.7 & 35.5 & 35.0 & 22.4 & 35.0 & 22.4 & 22.4 & 31.0 & 22.4 & 50.4 & 36.7 \\
\hline q4 & 33.8 & 36.1 & 33.8 & 21.2 & 32.7 & 21.2 & 21.2 & 33.8 & 21.2 & 38.4 & 36.1 \\
\hline q5 & 66.5 & 72.2 & 64.8 & 69.9 & 69.9 & 64.8 & 64.2 & 64.8 & 60.2 & 69.9 & 65.9 \\
\hline q6 & 63.1 & 67.1 & 72.2 & 64.8 & 67.1 & 72.2 & 70.5 & 72.2 & 61.3 & 70.5 & 69.4 \\
\hline q7 & 67.6 & 51.0 & 51.0 & 63.6 & 51.0 & 51.0 & 59.0 & 52.2 & 58.5 & 52.2 & 63.6 \\
\hline q8 & 68.8 & 72.8 & 72.8 & 68.8 & 72.8 & 58.5 & 54.5 & 61.9 & 58.5 & 66.5 & 56.2 \\
\hline r1 & 80 & 100 & 100 & 80 & 100 & 70 & 100 & 100 & 100 & 90 & 100 \\
\hline r2 & 120 & 130 & 130 & 120 & 130 & 130 & 150 & 130 & 120 & 110 & 130 \\
\hline r3 & 140 & 130 & 130 & 140 & 130 & 130 & 130 & 130 & 140 & 110 & 130 \\
\hline r4 & 80 & 80 & 90 & 90 & 80 & 80 & 90 & 90 & 120 & 90 & 90 \\
\hline r5 & 80 & 100 & 80 & 90 & 80 & 80 & 80 & 80 & 90 & 80 & 100 \\
\hline r6 & 100 & 140 & 90 & 100 & 90 & 90 & 90 & 90 & 100 & 90 & 140 \\
\hline r7 & 100 & 130 & 80 & 100 & 110 & 80 & 110 & 80 & 110 & 80 & 120 \\
\hline r8 & 80 & 90 & 80 & 80 & 80 & 80 & 80 & 90 & 80 & 80 & 90 \\
\hline
\end{tabular}

Table 5. Designs from the Pareto optimal front.

Due to difficulty in plotting results with respect to three orientations, results have been provided for three fixed yaw orientations $(\phi)$ such as $-20^{\circ},+20^{\circ}$ and $0^{\circ}$. 


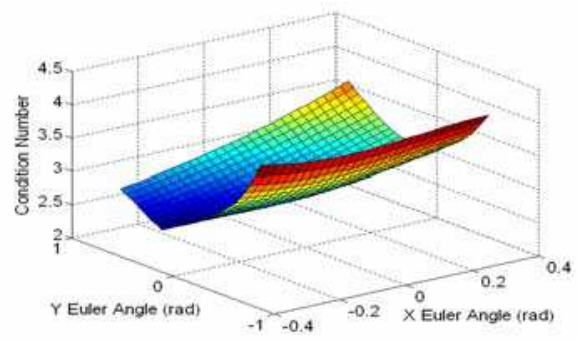

Fig. 8. Condition number vs. orientation at $\left(\phi=-20^{\circ}\right)$

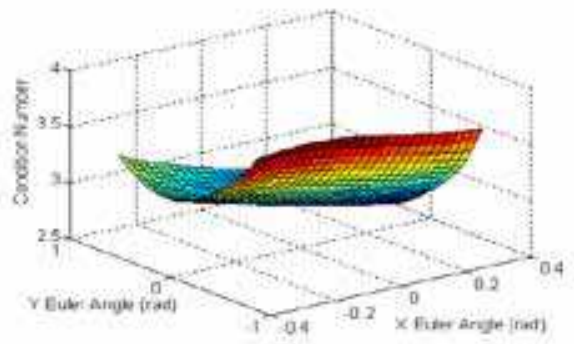

Fig. 9. Condition number vs. orientation at $\phi=20^{\circ}$

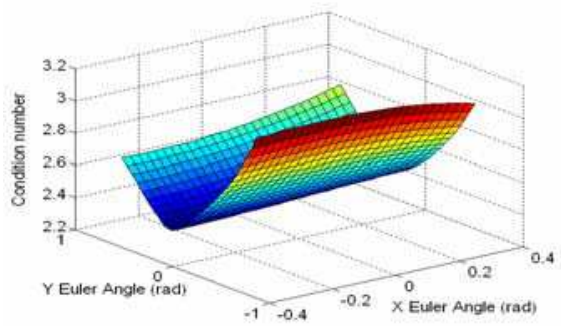

Fig. 10. Condition number vs. orientation at $\left(\phi=0^{\circ}\right)$

It is evident from the results shown (Figures 8-10) that the condition number is a small number close to the center of rotation and increases steadily when the manipulator is moved to the workspace extremities. Further it is also apparent from these figures that the condition number variation in the neighborhood is also smooth and the workspace is relatively free from the singularities. 


\section{Conclusion and Future Work}

A wearable soft parallel robot for ankle joint rehabilitation has been proposed after carefully studying the complexities of human ankle joint and its motions. The proposed device is an improvement over existing robots in terms of simplicity, rigidity and payload performance. The proposed device is very light in weight (total weight is less than $2 \mathrm{Kg}$ excluding the weight of support mechanism) and is inexpensive. The kinematic and workspace study is carried out and the performance indices to evaluate the robot design are discussed in detail. It is highlighted that to exploit the potential of parallel manipulators and overcome their shortcomings their design should be optimized. Three important criteria, the global condition number, the workspace volume and the norm of the actuator forces, are considered to evaluate the robot design. A multi-criteria, multi-variable optimization problem is formulated and the suitable constraints are defined. The feasibility of genetic algorithms to solve such multi-criteria optimization problem is emphasized and a brief discussion on existing techniques is also provided. We have attempted to use an algorithm which maximizes a fitness function using weighted formula approach and at the same time provides us with Pareto optimal solutions. The results obtained are discussed and several important inferences are drawn which will be helpful for the future course of this research. After carefully studying the proposed algorithm it was revealed that the possibilities of finding better solutions compared to the one presented in this chapter can not be ruled out. The present algorithm improves solutions in the direction of improving fitness of the aggregate function and does not attempt to improve the dominating solutions. Hence it will be interesting to use existing multi-objective evolutionary algorithms such as VEGA (Vector Evaluated Genetic Algorithms) and NSGA-II (Non-dominated Sorting Genetic Algorithms) which attempt to improve the dominated solutions. We have adopted GA parameters which are normally used in the literature, it will be interesting to change these parameters and study their effects on the convergence of our algorithm. Further, the Pareto solutions pose a difficulty in making a right choice from available alternate solutions. It is proposed to use Fuzzy Inferencing to assist the designer in making post Pareto decision.

It is evident from the results that the optimal design is left-right symmetric and hence connection points on the left and right side of the platforms can be considered to be the mirror images. In light of the symmetric design, it is proposed that while experimenting with newer algorithms in future, the reduced number of variables may be used.

\section{Acknowledgement}

The work is supported by Faculty of Engineering, Research and Development Fund, University of Auckland, New Zealand. 


\section{References}

Alp B. \& Agrawal S. K. (2002). Cable suspended robots: Design, planning and control, Proceedings of 2002 IEEE International Conference on Robotics and Automation, 42754280, 10504729 (ISSN), Washington, D.C., May 11-15, 2002.

Boudreau, R. \& Turkkan, N. (1996). Solving forward kinematics of parallel manipulators with a genetic algorithm. Journal of Robotic Systems, Vol. 13, No. 2, 111-125.

Coello C. A. C. and Lamont G. B. (2002). Applications of multi-objective evolutionary algorithms, World Scientific, 981-256-106-4, Singapore.

Dai J. S.; Zhao T. \& Nester C. (2004). Sprained ankle physiotherapy based mechanism synthesis and stiffness analysis of a robotic rehabilitation device, Autonomous Robots, Vol. 16, 207-218.

Deb K. (2005). Optimization for engineering design, algorithms and examples, Prentice Hall of India, 81-203-0943-X, New Delhi.

Deshmukh G. \& Michael P. (1990). A modified Powell method for six-degrees-of-freedom platform kinematics, Computers and Structures, Vol. 34, No. 3, 485-491.

Deutsch E. J.; Lewis J. A. \& Buedea G. (2007). Technical and patient performance using a virtual reality-integrated telerehabilitation system: preliminary finding, IEEE Transactions on Neural systems and Rehabilitation Engineering, Vol. 15, No.1, 30-35.

Dul J. \& Johnson G.E. (1985). A kinematic model of the human ankle, Journal of Biomedical Engineering, Vol. 7, 137-143.

Geng Z. \& Haynes L. (1991). Neural network solution for the forward kinematics problem of a Stewart platform, Proceedings of the IEEE International Conference on Robotics and Automation, 2650-2655, Sacramento, California.

Girone M. J.; Burdea G. C. \& Bouzit M. (1999). The Rutgers ankle orthopedic rehabilitation interface, ASME, Dynamic Systems and Control Division (Publication) DSC, Vol. 67, 305-312.

Girone M. J.; Burdea G. C., Bouzit M. \& Popescu V. (2000). Orthopedic rehabilitation using the Rutgers ankle interface, Studies in health technology and informatics, Vol. 70, 89-95.

Hassan M. \& Khajepour A. (2008). Optimization of actuator forces in cable-based parallel manipulators using convex analysis, IEEE Transactions on Robotics, Vol. 24, No. 3, 736-740.

Hesse S.; Schmidt H., Werner C. \& Bardeleben A. (2003). Upper and lower extremity robotic devices for rehabilitation and for studying motor control. Current Opinion in Neurology, Vol. 16, No. 6, 705-710.

Innocenti C. \& Parenti C. V. (1990). Direct position analysis of the Stewart platform mechanism, Mech. Mach. Theory, Vol. 25, No. 6, 611-621.

Krebs H. I. (2003) Rehabilitation robotics: performance-based progressive robot- assisted therapy, Autonomous Robots , Vol. 15, No. 1, 7-20.

Khatami S. \& Sassani F. (2002). Isotropic design optimization of robotic manipulators using a genetic algorithm method, Proceedings of the 2002 IEEE International Symposium on Intelligent Control, 562-567, Vancouver, October 27-30, Canada.

Knuth D. E. (2000). The art of computer programming: Vol.2, Addison-Wesley, 0201896834, Reading, Massachusetts.

Lemay J. \& Notash L. (2004). Configuration engine for architecture planning of modular parallel robots, Mechanism and Machine Theory, Vol. 39, No. 1, 101-117. 
Li L.; Zhu Q. \& Xu L. (2007). Solution for forward kinematics of 6-dof parallel robot based on particle swarm optimization, In Proceedings of the IEEE International Conference on Mechatronics and Automation, 2968-2973. Harbin, China.

Liu G.; Gao J, Yue H, Zhang X \& Lu G (2006). Design and kinematics analysis of parallel robots for ankle rehabilitation, Proceedings of the International Conference on Intelligent Robots and Systems, October 9 - 15, Beijing, China.

Liu X. J. (2006). Optimal kinematic design of a three translational DoFs parallel manipulator, Robotica, Vol. 24, 239-250.

Merlet J. P. (2003). Determination of the optimal geometry of modular parallel robots, Proceedings of the 2003 IEEE International Conference on Robotics \& Automation, 11971202, September 14-19, Taipei, Taiwan.

Nam Y. J. \& Park M. K. (2004). Geometric approach to forward kinematics of casing oscillator, Proceedings of Annual Conference of IEEE Industrial Electronics Society, 25822587, Busan, Korea.

Oyama E.; Maeda T., Tachi S., MacDorman K. F. \& Agah A. (2002). On the use of forward kinematics model in visually guided hand position control analysis based on ISLES model, Neuro computing, Vol. 44-46, 965-972.

Parenteau C. S.; Viano D.C. \& Petit P.Y. (1998). Biomechanical properties of human cadaveric ankle-subtalar joints in quasi-static loading. Journal of Biomechanical Engineering, Vol.120, No. 1, 105-111.

Pusey J. L.; Fattah A., \& Agrawal S. K. (2003). Design and workspace analysis of a 6-6 cablesuspended parallel robot, Mechanism and Machine Theory, Vol.39, No. 7, 761-778.

Sadjadian H. \& Taghirad H. D. (2005). Comparison of different methods for computing the forward kinematics of a redundant parallel manipulator. Journal of Intelligent and Robotic Systems, Vol. 44, No. 3, 225-246.

Schaffer J. D. (1985) Multiple objective optimization with vector evaluated genetic algorithms, Proceedings of the first international conference on Genetic Algorithms, 93100, Lawrence, Erlbaum.

Sergiu S.; Maties V. \& Balan R. (2006). Optimization of workspace of a 2 DOF parallel robot, Proceedings of the 2006 IEEE, International Conference on Mechatronics and Automation, 165-170, June 25 - 28, 2006, Luoyang, China.

Siegler S.; Chen J. \& Schneck C. D. (1988). The three-dimensional kinematics and flexibility characteristics of the human ankle and subtalar joints- Part I: Kinematics, Journal of Biomechanical Engineering, Vol. 110, 364-373.

Stock M. \& Miller K. (2003). Optimal kinematic design of spatial parallel manipulators: application to linear delta robot, Transactions of ASME, Vol. 125, 292-301.

Stump E. \& Kumar V. (2006). Workspaces of cable-actuated parallel manipulators, Journal of Mechanical Design ASME, Vol. 128, 159-167.

Su X. Y.; Duan B. Y. \& Zheng C. H. (2001). Genetic design of kinematically optimal fine tuning Stewart platform for large spherical radio telescope, Mechatronics, Vol. 11, 821-835.

Tsai L. W. \& Joshi S. (2000). Kinematics and optimization of a spatial 3-UPU parallel manipulator, Journal of Machine Design, Vol. 122, 439-446.

Yee C. S. \& Lim, K. B. (1997). Forward kinematics solution of stewart platform using neural networks, Neuro computing, Vol. 16, 333-349. 
Yoon J.; Ryu J. \& Lim K. B. (2006). Reconfigurable ankle rehabilitation robot for various exercises, Journal of Robotic Systems , Vol. 22 (Supplement), S15-S33, 2006. 


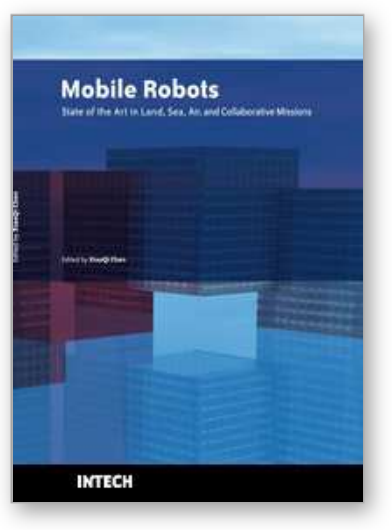

\author{
Mobile Robots - State of the Art in Land, Sea, Air, and \\ Collaborative Missions \\ Edited by XiaoQiChen
}

ISBN 978-953-307-001-8

Hard cover, 335 pages

Publisher InTech

Published online 01, May, 2009

Published in print edition May, 2009

Since the introduction of the first industrial robot Unimate in a General Motors automobile factory in New Jersey in 1961, robots have gained stronger and stronger foothold in the industry. In the meantime, robotics research has been expanding from fix based robots to mobile robots at a stunning pace. There have been significant milestones that are worth noting in recent decades. Examples are the octopus-like Tentacle Arm developed by Marvin Minsky in 1968, the Stanford Cart crossing a chair-filled room without human assistance in 1979, and most recently, humanoid robots developed by Honda. Despite rapid technological developments and extensive research efforts in mobility, perception, navigation and control, mobile robots still fare badly in comparison with human abilities. For example, in physical interactions with subjects and objects in an operational environment, a human being can easily relies on his/her intuitively force-based servoing to accomplish contact tasks, handling and processing materials and interacting with people safely and precisely. The intuitiveness, learning ability and contextual knowledge, which are natural part of human instincts, are hard to come by for robots. The above observations simply highlight the monumental works and challenges ahead when researchers aspire to turn mobile robots to greater benefits to humankinds. This book is by no means to address all the issues associated mobile robots, but reports current states of some challenging research projects in mobile robotics ranging from land, humanoid, underwater, aerial robots, to rehabilitation.

\title{
How to reference
}

In order to correctly reference this scholarly work, feel free to copy and paste the following:

P. K. Jamwal, S. Q. Xie, K. C. Aw and Y. H. Tsoi (2009). Multi-Criteria Optimal Design of Cable Driven Ankle Rehabilitation Robot, Mobile Robots - State of the Art in Land, Sea, Air, and Collaborative Missions, XiaoQiChen (Ed.), ISBN: 978-953-307-001-8, InTech, Available from:

http://www.intechopen.com/books/mobile-robots-state-of-the-art-in-land-sea-air-and-collaborativemissions/multi-criteria-optimal-design-of-cable-driven-ankle-rehabilitation-robot

\section{INTECH}

open science | open minds

\section{InTech Europe}

University Campus STeP Ri

Slavka Krautzeka 83/A

51000 Rijeka, Croatia

Phone: +385 (51) 770447

\section{InTech China}

Unit 405, Office Block, Hotel Equatorial Shanghai

No.65, Yan An Road (West), Shanghai, 200040, China

中国上海市延安西路65号上海国际贵都大饭店办公楼 405 单元

Phone: +86-21-62489820 
Fax: +385 (51) 686166

Fax: +86-21-62489821

www.intechopen.com 
(C) 2009 The Author(s). Licensee IntechOpen. This chapter is distributed under the terms of the Creative Commons Attribution-NonCommercialShareAlike-3.0 License, which permits use, distribution and reproduction for non-commercial purposes, provided the original is properly cited and derivative works building on this content are distributed under the same license. 